\title{
S. Martin, Voix et relation. Une poétique de l'art littéraire où tout se rattache
}

\section{Germana Berlantini}

\section{(2) OpenEdition}

1 Journals

\section{Edizione digitale}

URL: http://journals.openedition.org/studifrancesi/10634

DOI: 10.4000/studifrancesi.10634

ISSN: 2421-5856

\section{Editore}

Rosenberg \& Sellier

\section{Edizione cartacea}

Data di pubblicazione: 1 décembre 2017

Paginazione: 601-602

ISSN: 0039-2944

\section{Notizia bibliografica digitale}

Germana Berlantini, «S. Martin, Voix et relation. Une poétique de l'art littéraire où tout se rattache», Studi Francesi [Online], 183 (LXI | III) | 2017, online dal 01 février 2018, consultato il 22 janvier 2021. URL: http://journals.openedition.org/studifrancesi/10634; DOI: https://doi.org/10.4000/studifrancesi. 10634

Questo documento è stato generato automaticamente il 22 janvier 2021.

\section{(c)}

Studi Francesi è distribuita con Licenza Creative Commons Attribuzione - Non commerciale - Non opere derivate 4.0 Internazionale. 


\title{
S. Martin, Voix et relation. Une poétique de l'art littéraire où tout se rattache
}

\author{
Germana Berlantini
}

\section{NOTIZIA}

SERGE MARTIN, Voix et relation. Une poétique de l'art littéraire où tout se rattache, Taulignan, Éditions Marie Delarbre, Collection Théories, 2017, 356 pp.

1 Il saggio si propone come un itinerario critico attraverso luoghi scelti della teoria contemporanea e delle opere letterarie al fine di ricostruire, per suggestioni e senza intento di sistematicità, la trama degli scambi fra le due nozioni della voce e della relazione. Nozioni che il critico non intende presentare come oggetti di ricerca circoscritti o metodologie specifiche ma all'intersezione fra campi del sapere, fenomeni e livelli di astrazione molteplici. Come argomenta infatti Serge MARTIN, nella misura in cui si concepisca il lavoro di concettualizzazione come attività di 'dissociazione', intersezione, mobilitazione di rapporti reticolari operata a partire dall'unità di nozioni semplici, il carattere comune, ambiguo o quotidiano di tali oggetti di partenza può costituire un efficace antidoto contro il rischio di tecnicismi ingannevoli. Assumendo come postura epistemologica la distinzione fra specialismo e studio dello specifico, il saggio si sviluppa senza soluzione di continuità secondo il movimento di un originale stile argomentativo, quello di una ricerca 'allo stato nascente', la quale, respingendo il paradigma narratologico come struttura progressiva e finalizzante della scrittura teorica, produca movimenti concettuali senza approdo. Sul solco di quello che Henri Meschonnic ha definito l'effetto critico, il volume procede secondo un percorso a spirale che, assumendo l'incompiutezza come forma del pensiero, fa di ogni nodo tematico e di ogni problema critico l'occasione di un nuovo inizio, in un'inesausta interrogazione dei propri presupposti. L'itinerario proposto non ambisce dunque a delimitare per accerchiamento progressivo un dominio corrispondente all'insieme di manifestazioni 
fenomeniche dei due termini in esame, quanto piuttosto ad aprire, ogni volta di nuovo, degli spazi empirici di 'gioco' fra di esse, secondo l'andamento aperto e problematico di un'autentica "chercherie".

2 Così, dopo una breve introduzione («La voix, la relation, une aventure critique», pp. 7-17) e rifiutandosi a qualunque definizione preliminare, la prima sezione del saggio («Chercher la voix, chercher la relation», pp.127-316) immerge il lettore in una concatenazione di letture, problematizzazioni, questionamenti in rapporto dialogico con un corpus di teoresi contemporanea (da Laurent Jenny a Sylvie Patron, da Gabriel Bergounioux a Gilles Philippe) dalle quali ciascuno potrà ricavare le proprie, operative, costellazioni di usi e significati relativi alle nozioni di voce e relazione. 0 , per meglio dire, di voce-relazione, nella misura in cui Martin si sforza di articolare il problema della "voix" quanto più lontano possibile dalla sua riduzione a un "effet de voix" e a una soggettività singolare. Sul solco delle analisi condotte da Meschonnic in Critique du rythme, lo studioso fonda infatti il proprio discorso teorico su tre pilastri critici: il dialogismo intrinseco della poesia, la relazionalità costitutiva del soggetto e il paradigma di una relazione di relazione. Per cui se è vero che non esiste scrittura dell'"io" che non declini contemporaneamente una propria forma del "tu", secondo la reciprocità specifica della persona umana, per Martin non si tratta tanto di far posto a un ritorno del soggetto nello spazio letterario quanto piuttosto di pensare i modi processuali di una soggettivazione, quella delle opere, sempre e imprescindibilmente relazionale dunque sociale, storica, collettiva. In continuità con Meschonnic, Martin assume dunque che i fatti letterari richiedano come chiave d'accesso un'antropologia critica del linguaggio la quale, unificando il campo dell'umano nello spazio di un corpolinguaggio, superi le antinomie tradizionali di interiorità ed espressività, individuale e sociale. In questa prospettiva la centralità della voce discende dal suo ruolo di frontiera che non divide bensì unifica: nello spazio di ascolto che essa è, tutti i dualismi concettuali vengono meno, fino a rendere insignificante la stessa opposizione di autore e lettore. Parlare di voce-relazione, articolare cioè non tanto una struttura a due termini quanto il rapporto che fra questi intercorre, uno spazio di continuità e di reciprocità indefinitamente intensificabile (in questo senso Martin parla di relazione di relazione) significa dunque procedere a una "échoïsation" della voce che si scopre essere passaggio di voce, internamente rifratta e pluralizzata. Attraversando le problematiche critiche dell'endofasia, del tono, dell'eloquenza, dello stile, del fraseggio, analizzando la produzione teorica contemporanea col fine di demistificarne il 'riduzionismo', lo 'storicismo' e il regime binario di differenziazione fra linguaggio e letteratura, oralità e scrittura, sociale e individuale, norma linguistica ed espressività, Martin giunge a presentare il poema come forma presente di tale voce-relazione.

3 Alla seconda parte del volume - «Parcours de la voix-relation» (pp. 127-315) - è affidato il compito di manifestare tale qualità relazionale della voce nello spazio empirico di opere contemporanee scelte. All'andamento a spirale fin qui adottato, dove i capitoli sfociano l'uno nell'altro - energeia e mai ergon del concetto, come per la lingua in Humboldt -, si affianca una ripartizione per percorsi tematici. Così, se il problema della 'ripresa' come riscrittura, ripetizione, performance dà luogo a una lettura delle opere di Jean-Luc Parant e Charles Pennequin, quello del 'gesto' investe la parola di James Sacré la cui poesia è dal critico assimilata a un vero e proprio movimento relazionale, a un "geste parlé" che riduce al minimo il proprio quoziente di identificazione per massimizzare quello di interazione, suggestione, scambio-nel duplice senso di 
condivisione e antagonismo del potlatch di Marcel Mauss. Se la nozione di 'spazio' implica una tematizzazione dell'aperto' come ricerca di relazione attraverso tre esempi di scrittura per l'infanzia - Olga Lecaye, Nadja e Grégoire Solotareff -, quella di 'soffio', come forma del rapporto fra corpo e linguaggio diventa chiave per leggere i romanzi di Sylvie Germain, spazio di una voce unitaria e plurale al contempo. Infine Martin fa ritorno sul pensiero e l'opera di Henri Meschonnic mediante un itinerario in tre tappe: nel capitolo dedicato alla nozione di 'biografia', ricostruendone la singolare dialettizzazione di vivere e scrivere proposta a partire da Écrire Hugo; percorrendone il rapporto di prossimità e differenza col pensiero di Paul Ricœur in quello dedicato al 'viaggio'; con una più globale visione d'insieme del suo percorso critico e poetico nel capitolo finale significativamente intitolato "Commencements». Alla fine di questa parabola critica, dunque, la poesia (la letteratura fuori dal suo regime di definizione binario) può apparire come interazione fra una forma di linguaggio ed una forma di vita: né rispecchiamento né rappresentazione, in termini non marxisti né ermeneutici, tale rapporto è per Martin 'invenzione' e inestricabilità reciproca. Liberata dall'enucleazione del senso come dall'analisi dei suoi dispositivi teorici, la scrittura si rivela allora luogo di una prossimità di voci, risonanza di ascolti, continuità del poetico e del politico, del poetico e dell'etico. Di modo che-in una conclusione significativamente intitolata «Une voix 'où tout se rattache'» (pp. 317-323) - la vocerelazione dichiara la propria equivalenza col movimento di soggettivazione mediante il quale prende corpo, nel poema, un "je-tu" produttivo di un "je-histoire" e irriducibile a qualunque topica testuale. Il volume è completato da una bibliografia (pp. 325-340) e dagli indici (pp. 341-355). 